\title{
Inflammatory factors are crucial for the pathogenesis of post-traumatic osteoarthritis confirmed by a novel porcine model: "Idealized" anterior cruciate ligament reconstruction" and gait analysis
}

\section{Ruipeng Zhao}

Second Hospital of Shanxi Medical University

\section{Zhengquan Dong}

Second Hospital of Shanxi Medical University

Xiaochun Wei

Second Hospital of Shanxi Medical University

Xiaodong Gu

Bethune Hospital of Shanxi Medical University

\section{Pengfei Han}

Changzhi Medical College Affiliated Heping Hospital

Zhi Lv

Second Hospital of Shanxi Medical University

Hongru Wu

Shanxi Institute of Sports Science

\section{Yanxia Yan}

Second Hospital of Shanxi Medical University

\section{Lingan Huang}

Second Hospital of Shanxi Medical University

Haoqian Li

Second Hospital of Shanxi Medical University

Fei Li

Second Hospital of Shanxi Medical University

Pengcui Li ( $\square$ at19834513411@163.com )

Second Hospital of Shanxi Medical University

Research article 
Keywords: post-traumatic osteoarthritis, anterior cruciate ligament reconstruction, inflammation, gait analysis, minipig

Posted Date: March 4th, 2021

DOl: https://doi.org/10.21203/rs.3.rs-269041/v1

License: (c) (1) This work is licensed under a Creative Commons Attribution 4.0 International License. Read Full License 


\section{Abstract}

Background: The study was performed to determine whether idealized anterior cruciate ligament reconstruction (IACL-R) restores normal gait features, and whether inflammatory factors are involved in the pathogenesis of post-traumatic osteoarthritis (PTOA).

Methods: Fourteen mature female minipigs were allocated to a sham group $(\mathrm{n}=7)$ or an IACL-R group ( $\mathrm{n}$ $=7$ ). Load asymmetry during gait was recorded using a pressure-sensing walkway measurement system to evaluate the gait features of the right knee joint before and after surgery. Inflammatory factors (including interleukin [IL]-1a, IL-1 1 , IL-2, IL-6, IL-8, IL-18, tumor necrosis factor-a, and granulocytemacrophage colony-stimulating factor) in synovial fluid were measured using Luminex assays before and after surgery. Cartilage integrity and the subchondral bone plate of the right knee were evaluated using histology and imaging at 3 months postoperatively.

Results: Swing time and stance time returned to their preoperative values on day 31, while maximum force, contact area, peak force, and impulse returned to their preoperative values on day 45 after the surgery in the IACL-R group ( $P=0.073,0.053,0.107,0.052,0.152$, and 0.059 , respectively).Thus, IACL-R restored normal gait. Compared with their preoperative concentrations, all tested inflammatory factors showed significantly increased concentrations in the synovial fluid in the IACL-R group, especially at 3, 7, and 15 days postoperatively. X-ray, computed tomography, magnetic resonance imaging, and histological data showed severe cartilage damage in the IACL-R model.

Conclusion: IACL-R restored normal gait features but caused significant cartilage damage, indicating that significantly elevated inflammatory factors maybe crucial for the pathogenesis of PTOA.

\section{Background}

Post-traumatic osteoarthritis (PTOA) is considered to be a consequence of the biological and mechanical effects of a joint injury[1]. Anterior cruciate ligament $(A C L)$ rupture, one of the most common joint injuries in young people, is conventionally treated using surgical ACL reconstruction (ACL-R) with a graft to restore the biomechanical stability of the joint. However, even with the best surgical techniques available, these patients remain at a high risk for PTOA [2-5]. Although it is quite clear that both mechanics and biological factors affect PTOA, the relative contribution of each is unknown. A better understanding of the underlying mechanism may lead to the formulation of therapies that prevent PTOA

Recently, Han et al[6]. developed an "idealized" ACL autograft reconstruction (IACL-R) model in minipigs. In this model, the femoral attachment of the native ACL is cored out and immediately reattached as an ideal graft to restore the normal biomechanics of the joint. Surprisingly, the authors found that cartilage degeneration still occurred despite this reconstruction. However, the authors could not conclusively prove that their IACL-R model fully restored joint kinematics as compared to ACL-intact control knees.

Additionally, it was not possible to elucidate the precise inflammatory changes that occurred in the IACL-R model, especially in the "golden stage," i.e. the early postoperative period. Therefore, the purpose of the 
present study was to clarify the gait characteristics and roughly or indirectly evaluate the changes in joint kinematics in the IACL-R model by using gait analysis, as well as to evaluate the early changes in inflammatory factors and assess their role in the pathogenesis of PTOA. Furthermore, cartilage integrity and the subchondral bone plate were evaluated using imaging and histological examination. We hypothesized that the IACL-R model can functionally restore joint gait features, and that a significant increase in inflammatory factors triggers cartilage degradation in this model.

\section{Methods}

\section{Study Design}

All procedures for animal research in this study were approved by the Ethics Committee of the Second Hospital of Shanxi Medical University (no. 2017046). All minipigs were purchased from the Beijing Shichuang Century Minipig Breeding Base (Certificate number: SCXX(jing)2013-0008). Fourteen mature female minipigs (age, $18 \pm 1.58$ months; weight, $42.8 \pm 3.97 \mathrm{~kg}$ ) were randomized to two groups based on animal ear numbers: an IACL-R group $(n=7)$ and a sham group $(n=7)$. Unilateral surgery was performed on the right hind limbs (RHs) of all minipigs. All animals were housed individually in adjacent pens at the China Institute for Radiation Protection (Certificate number: SYXK(Jin)2016-0002). All animals were euthanized with a pentobarbital overdose at 3 months after the surgery. Specific information on animal care can be found in Additional text (Text A).All subjective scores were independently completed by two experienced examiners, who were blinded to the animal number and experimental condition

\section{Surgical Technique}

The specific operation in the IACL-R group was performed as described by Han et al[6] (Fig 1).The minipigs in the sham group underwent a similar arthrotomy procedure with temporary patellar dislocation.

\section{Quadruped Gait Assessment}

Before testing, the Tekscan operating system software was equilibrated and calibrated[7]. Six gait indicators related to biomechanics, namely, maximum force, contact area, peak force, impulse, stance time, and swing time, were determined [8-10]. We carefully checked each image (frame) to ensure the accuracy of the results (Additional Fig 1). To rule out differences in learning skills and walking states between the two groups of minipigs, each animal was subjected to over 10 successful training sessions per day for 10 consecutive days before the surgery, and all indicators were expressed as a ratio of the average values for the left hind limb (LH) divided by the average values for the $\mathrm{RH}$. Gait data were collected preoperatively (day 0 ) and postoperatively on days $8,16,31,45,61,75$, and 90 . All the results are the average values of 5 successful repeated walkway trials performed at each time point for each animal.

\section{Synovial Fluid Collection}


Synovial fluid from the $\mathrm{RH}$ of all the animals were collected preoperatively (day 0 ) and postoperatively on days $3,7,15,30,60$ and 90 . Preoperatively, synovial fluid lavages were collected after anesthesia. $2 \mathrm{ml}$ of isotonic saline solution was injected intra-articularly using a sterile syringe inserted through the inferior patellar tendon. The knee was then manually cycled through flexion and extension twenty times to distribute the fluid within the joint before collection via joint aspiration[11].. Postoperatively $\llbracket$ synovial fluid was directly collected after anesthesia induction.

\section{Luminex Assay}

The Millipore Porcine Cytokine Magnetic Bead Panel (EMD Millipore, No. PCYTMAG-23K) was used to measure the levels of interleukin [IL]-1a, IL-1 $\beta$, IL-2, IL-6, IL-8, IL-18, tumor necrosis factor-a(TNF-a), and granulocyte-macrophage colony-stimulating factor( GM-CSF). Luminex assay as described previously ${ }^{6}$.

\section{Imaging Assessment}

At 3 months after the surgery, the minipigs were euthanized with a pentobarbital overdose, and their RHs were severed from the hip joint. Each RH was immediately subjected to X-ray examination, computed tomography (CT), three-dimensional CT reconstruction (3D CT), and magnetic resonance imaging (MRI). The specific imaging parameters are listed in the Additional text (Text B).

We determined the Kellgren-Lawrence grade of the $\mathrm{RH}$ of each animal by examining the X-ray film[12, 13]. The CT values and thickness of the subchondral bone plate were determined[14]. To avoid interference by metal artifacts, we obtained CT scans in only the middle plane of sagittal from medial femoral condyle (MFC) and medial tibial plateau (MTP) of the RH. We also determined the whole-organ MRI score (WORMS) of the MFC and MTP of the RH[15, 16]. The ordinal scores ranged from 0 (normal thickness, no signal enhancement) to 6 (diffuse full-thickness defect, $>75 \%$ of the area), and the WORMS sum ranged from 0 to 12 .

\section{Macroscopic Cartilage and Osteophyte Assessment}

Macroscopic damage to the articular cartilage surfaces and osteophyte formation on the MFC, MTP, lateral femoral condyle (LFC), lateral tibial plateau (LTP), and trochlea were scored according to the Osteoarthritis Research Society International (OARSI) recommendations for the sheep and goats[17].

\section{Histological Assessment}

Histological samples were obtained by drilling ( $\varphi 8 \mathrm{~mm}$; MOC Medizinische Gerate Gmbh, Fedderingen, Germany) the middle region of the MTP. The tissue sections were stained with safranin 0 and fast green to evaluate the cartilage using the OARSI guidelines [17].

\section{Immunohistochemical Assessment}


Tissue sections from the MTP were used to detect the distribution of type II collagen (Col-II) and matrix metalloproteinase (MMP)-13 in the cartilage. Specific antibodies against Col-II (ab34712, Abcam) and MMP-13 (K009743P, Solarbio) were used. The detailed immunohistochemical procedure has previously been described[18].

\section{Statistical Analysis}

SPSS v13.0 statistical software was used to analyze the collected data. Differences in gait and inflammatory factor levels between the preoperative (day 0 ) and postoperative time points in the same group were analyzed using t test-paired. Differences in gait, inflammatory factors, CT values and thickness of the subchondral bone plate ,T2 values at the same time point between the sham and IACL-R groups were estimated using the two-group $t$ test-unpaired. Differences in macroscopic cartilage and osteophyte scores, microscopic cartilage scores, and WORMS between the sham group and IACL-R group were estimated using nonparametric tests (Wilcoxon rank sum test). Differences in Kellgren-Lawrence grades between the sham and IACL-R groups were estimated using Fisher probabilities. Statistical significance was determined at $\mathrm{P}<0.05$.

\section{Results}

Total weight gain during the 90-day study period was similar between the sham $(6.3 \pm 2.26 \mathrm{~kg})$ and IACL$R(5.87 \pm 2.43 \mathrm{~kg})$ groups $(P=0.351)$. All animals recovered from the anesthesia and were fully awake within $4 \mathrm{~h}$ after the surgery. Humane endpoints did not occur during this study. There were no instances of infection or death occurred outside of planned euthanasia or humane endpoints.

\section{Quadruped Gait Analysis}

In the sham group, the values of swing time $(P=0.051)$ from day 8 until euthanasia did not significantly differ from their values on day 0 . Similarly, the values of contact area $(P=0.051)$, and stance time $(P=$ $0.050)$ from day 16 until euthanasia did not significantly differ from their values on day 0 . The values of maximum force $(P=0.051)$, peak force $(P=0.194)$ and impulse $(P=0.055)$ from day 31 until euthanasia did not significantly differ from their values on day 0 (Fig 2; Additional Table 1).

In the IACL-R group, the ratios of all gait parameters of the LH to those of the RH initially showed an increasing trend, then a decreasing trend, and finally, an increasing trend again. The values of swing time on day $16(P=0.073)$ did not differ from their values on day 0 . The values of stance time $(P=0.053)$ on day 31 did not differ from their values on day 0 . The values of maximum force $(P=0.107)$, contact area $(P=0.052)$, peak force $(P=0.152)$, and impulse $(P=0.059)$ on day 45 did not differ from their values on day 0 . These findings indicated that the above gait parameters returned to their normal status at the specified time points. However, on day 75 , the parameters maximum force $(P=0.002)$, contact area $(P<$ $0.001)$, peak force $(P=0.021)$, impulse $(P<0.001)$, and stance time $(P=0.002)$ showed a deteriorating trend. Significant differences in their values, as compared to day 0 , began to appear again and these differences persisted until the animals were euthanized. (Fig 2; Additional Table 1). 
The gait parameters of the $\mathrm{LH}$ and $\mathrm{RH}$ were similar under healthy conditions (day 0 ) for symmetric. The $\mathrm{LH}: \mathrm{RH}$ ratios of all gait parameters, were similar and close to 1 , and did not significantly differ between the sham and the IACL-R groups before the surgery. While the postoperative ratios of the gait parameters were significantly greater than 1 in both groups, the values in the IACL-R group were significantly greater than those in the sham group, especially on days 8 and 16 . The $\mathrm{LH}: \mathrm{RH}$ ratio of the gait parameters was close to 1 on days 45 and 61 , indicating that there were no significant differences between the two groups. On day 75, this ratio began to rise in the IACL-R group but remained constant in the sham group, and a significant difference again appeared between the two groups. On day 90 , all gait parameters other than swing time significantly differed between the sham and IACL-R groups (Fig 2; Additional Table 1).

\section{Inflammatory Factors Analysis}

In the sham group, the concentrations of inflammatory factors (IL-1 $\mathrm{a} \bigotimes \mathrm{P}=0.111 \mathrm{X}, \mathrm{IL}-1 \beta \bigotimes \mathrm{P}=0.055 \mathrm{~V}, \mathrm{IL}-6$ $\triangle P=0.069 \rrbracket, I L-8 \rrbracket P=0.369 \rrbracket$, and $T N F-a \rrbracket P=0.087 \rrbracket)$ from day 30 until euthanasia did not significantly differ

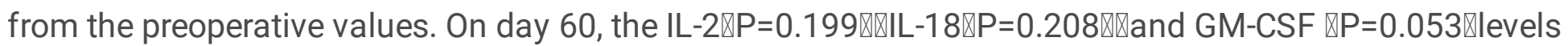
did not significantly differ from their preoperative values (Fig 3).

In the IACL-R group, the concentrations of all inflammatory factors, except for IL-18, showed a dramatically increasing trend and then a decreasing trend. The IL-8 concentration showed a significant rebound from day 60 to 90 . The IL-18 level progressively increased after the surgery until euthanasia, and thus, peaked on day 90 . The concentrations of all inflammatory factors from day 3 until euthanasia significantly differed from their preoperative values. The IL-1 a $(P<0.001) \mathbb{\text { IL }}-6(\mathrm{P}<0.001)$ \and IL-8 $(P<0.001)$ concentrations peaked on day 3 ; the IL-1a $(P<0.001), I L-1 \beta(P<0.001)$, and GM-CSF $(P<0.001)$ concentrations peaked on day 7 , while the IL-2 $(P<0.001)$ and TNF-a $(P<0.001)$ concentrations peaked on day 15 . These results suggest that the levels of inflammatory factors increase immediately after joint trauma, and thus, preventive anti-inflammatory strategies should be adopted as soon as possible (Fig 3).

On day 0 , the concentrations of all the inflammatory factors in the sham group and the IACL-R group were very low, with no significant between-group difference. After the surgery, although the trends of the inflammatory factor concentrations in the two groups were similar, the increase was more obvious in the IACL-R group. At all time points after the surgery, the concentrations of all inflammatory factors were significantly higher in the IACL-R group than in the sham group. (Fig 3).

\section{Imaging Assessment}

In the IACL-R group, X-ray examinations showed the joint spaces were not clearly defined. Furthermore, mild osteophyte formation was observed. Occasionally, narrowing of the joint space, especially in the medial compartment, was seen (Fig 4a-4d). The Kellgren-Lawrence grades did not significantly differ between the sham and IACL-R groups ( $P=0.132 ;$ Table 1 ). The CT values (water: 0 , bone: 1000 ) of the 
subchondral bone plate at the MFC $(P<0.001)$ and MTP $(P<0.001)$ were significantly higher in the sham group than in the IACL-R group (Fig 5a). The thickness of the subchondral bone plate at the MFC ( $P=$ 0.051) and MTP ( $P=0.023)$ was higher in the sham group than in the IACL-R group (Fig $5 \mathbf{b})$. Threedimensional CT reconstruction showed that all joint surfaces were smooth and flat in the sham group, whereas in the IACL-R group, the joint surfaces, especially the patellofemoral surface, were rough and uneven. (Fig 4e-4h).

In the sham group, MRI OSag-fs PD showed that the cartilage layer was intact, continuous, and uniform in thickness and signal intensity. In contrast, in the IACL-R group, the cartilage continuity was poor, with obvious local defects and signal variations, especially, in the middle plane (Fig 4i, 4k). The WORMS values of the MFC $(P=0.015)$ and MTP $(P=0.049)$ were higher in the IACL-R group than in the sham group (Table 2, Fig 5c-5e). Osag T2MAP showed that the articular cartilage was intact and relatively uniform in color (orange) in the sham group. In the IACL-R group, the cartilage showed poor continuity, as indicated by the focally mixed, irregular color and blue-green plaques (Fig 4j, 4I). The T2 values of the MFC $(P<0.001)$ and MTP $(P<0.001)$ were significantly higher in the IACL-R group than in the sham group (Fig 5f).

\section{Macroscopic Cartilage and Osteophyte Assessment}

All the animals in the IACL-R group showed macroscopic degeneration of the articular surfaces. Large erosions down to the subchondral bone were observed occasionally (Fig 6). Finally, the macroscopic cartilage scores of the MFC $(P=0.008)$, LFC $(P=0.023)$, MTP $(P=0.038)$, LTP $(P=0.036)$, trochlea $(P=0.003)$ and sum $(P=0.004)$ were significantly higher in the IACL-R group than in the sham group.. (Table 2, Additional Fig 2a-2f).

The edges of the MFC, LFC, MTP and LTP were smooth in both the sham and IACL-R groups. Mild irregular protrusions were found on both sides of only the trochlea in the IACL-R group (Fig 6). Thus, the macroscopic osteophyte scores of the MFC ( $P=0.275)$, LFC ( $P=0.645)$, MTP $(P=0.645)$, and LTP $(P=0.591)$ did not differ between the IACL-R and sham groups. Only the score of the trochlea $(P=0.018)$ was significantly higher in the IACL-R group than in the sham group. Finally, the macroscopic osteophyte sum scores was significantly higher in the IACL-R group than in the sham group $(P=0.009)$ (Table 2, Additional Fig 2g-2l).

\section{Histological Assessment}

In the sham group, we observed a slight decrease in safranin 0 staining and slight surface fibrillation. In the IACL-R group, however, we observed a moderate increase in chondrocyte density, increased chondrocyte cloning, severely increased fibrillation, and occasional erosions to the subchondral bone (Fig 7a). The microscopic OARSI cartilage scores, both the sum scores $(P=0.004)$ and the scores for single 
indicators, other than demark score $\varangle \mathrm{P}=0.072 \varangle \llbracket$ showed that the cartilage damage was more severe in the IACL-R group than in the sham group (Table 2, Additional Fig 2m-2r).

Immunohistochemical staining showed that Col-II expression was significantly lower and MMP-13 expression was significantly higher in the IACL-R group than in the sham group. This is consistent with reduced osteoarthritis damage in the minipigs in the sham group (Fig 7b)

\section{Discussion}

PTOA is a degenerative disease of the articular cartilage caused by complex biomechanical and biochemical factors[1]. Some clinical evidence indicates that patients who undergo ACL-R are prone to developing symptoms of PTOA in the long term, despite have a relatively stable joint structure[19]. However, whether biochemical factors contribute to the development of PTOA after ACL-R is largely unknown since no effective animal model or precise experimental method was available so far. To independently evaluate the effects of inflammation on cartilage degeneration after joint injury, Han et al [6].developed the IACL-R model. Their IACL-R model was created using minipigs, which are commonly used in orthopedic studies, as they have considerable advantages [20-23]. In this study, the porcine model developed osteoarthritis, as noted on imaging and histology, at 3 months after IACL-R, despite the animals showing normalization of gait. Furthermore, Luminex assay showed high concentrations of many inflammatory factors in the IACL-R group after surgery, especially in the early stage. This suggests that inflammatory reaction may play a crucial role in the development of PTOA after ACL-R.

Gait analysis is a relatively sensitive test for abnormal biomechanics and pain in the knee joint[24-26]. The minipigs in the sham group underwent only arthrotomy and temporary patellar dislocation, which preserved the normal joint biomechanics. Therefore, the gait index quickly returned to normal by 16-31 days after the surgery. In the IACL-R group, although the joint-stabilizing structures were intact, the minipigs underwent IACL-R. The surgical trauma was greater, and the surgical legs were extremely maladaptive. This may have caused the maximum force of the surgical leg to significantly decrease by day 8. As a compensatory mechanism, the maximum force of the contralateral leg increased. This phenomenon is consistent with the changes seen in clinical practice[27]. Hence, the contralateral leg : surgical leg ratio was significantly greater than 1 on day 8 . With time, the pain in the surgical leg may have decreased with constant adaptive training. Then, the maximum force of the surgical leg gradually returned to normal, and the contralateral leg:surgical leg ratio was close to 1 on day 45 , with no significant difference from the ratio on day 0 . Furthermore, there was no significant difference in maximum force between the IACL-R and sham groups at this time. Therefore, we concluded that the IACL$\mathrm{R}$ model can restore the normal maximum force of the knee joint. This model can be used to independently study the inflammatory reaction associated with PTOA. It should however be noted that in the IACL-R group, the maximum force of the surgical leg continued to significantly decrease on days 75 and 90 . The reason may be that inflammatory factors triggered knee cartilage degeneration and induced more severe pain symptoms. The contact area of the surgical leg was the smallest on day 8 . It was possibly due to pain and inadaptation of the surgical leg. The trend of the changes in the contact area, 
peak force and impulse were similar to that of the changes in maximum force, possibly owing to essentially the same underlying reasons. The gait cycle consists of a stance phase and a swing phase[28, 29]. During the stance phase, the surgical leg was in a weight-bearing state and more affected. So, a significant difference in stance time, as compared to its preoperative value, appeared again on day 75. However, during the swing phase, the surgical leg was in a non-weight-bearing state and less affected. Therefore, the swing time of the surgical leg recovered faster, and no significant differences from the preoperative value were seen in this parameter from day 16 until euthanasia.

Inflammatory factors are tightly associated with the pathogenesis of PTOA[30]. This study is the first to focus on the changes in inflammatory factors in the early postoperative period in minipigs. Studies have shown that when activated, synovial cells, mononuclear macrophages, and articular chondrocytes secrete a variety of inflammatory factors, particularly IL-1[31]. Abnormally elevated IL-1 levels can induce chondrocytes to synthesize excessive nitric oxide, reduce proteoglycan synthesis, and accelerate chondrocyte apoptosis[32]. In addition, IL-1 can stimulate the secretion of matrix metalloproteinases and induce cartilage matrix degradation. Furthermore, IL-1 can stimulate synovial cell degeneration, proliferation, and secretion of other inflammatory factors[33]. The minipigs in the IACL-R group underwent IACL-R and the surgical trauma was greater. Therefore, all the tested inflammatory factors were significantly increased in the early postoperative period. Over time, the immune response was activated, granulation tissue continued to form, and the expression of inflammatory factors was significantly reduced, leading to the beginning of the chronic inflammation stage. Finally, the inflammatory factor levels dropped significantly, though IL-18 levels continued to rise after surgery until euthanasia. Our preliminary inference was that IL-18 may be positively correlated with the degree of articular cartilage degeneration. In summary, the elevated inflammatory factors may have interacted with each other, and ultimately triggered irreversible degradation of the articular cartilage.

The minipigs in the IACL-R group showed moderate osteoarthritis on imaging tests despite the restoration of normal joint gait features. These changes may be attributable to the severe inflammatory reaction in the knee joint. However, the Kellgren-Lawrence grades did not significantly differ between the IACL-R and sham groups. This result may be attributable to the assessment being carried out on disconnected and non-weight-bearing RHs, which could not truly reflect joint stenosis. In addition, at 3 months after the operation, the PTOA may still have been in an early stage. Changes in the microenvironment of the subchondral bone are important in osteoarthritis. Our findings are consistent with those of Burr and Sniekers $[34,35]$ in that the density and thickness of the subchondral bone plate were reduced in an animal model of osteoarthritis. Changes, including the decreases of the thickness and density of the subchondral bone plate and the increases of its porosity, promotes the invasion of blood vessels into the cartilage and the diffusion of inflammatory factors, accelerating the pathological circulation between bone and cartilage, and exacerbating the occurrence of osteoarthritis. MRI OSag-fs PD showed that the highest regions of the MFC and MTP showed more severe changes, which may be due to the fact that the articular surface was not covered by the meniscus and bore a greater force. Under the catalysis of inflammatory reactions, lesions developed first on the highest regions of the MFC and MTP and were consequently more severe, which is consistent with the results of long-term investigations in a porcine 
model[36]. The T2 value of Osag T2MAP is associated with free water content in cartilage tissue. The more severe the cartilage damage, the more free water and the higher the T2 value; thus, the T2 value is an objective and reliable marker[37]. The results of Osag T2MAP and their underlying causes were similar to those of OSag-fs PD.

The minipigs from the IACL-R group were found to have developed severe cartilage degeneration on histological examination, despite the restoration of normal joint biomechanics. The loss of GAGs and Coll-II was more severe in the IACL-R group than in the sham group. In contrast, the MMP-13 level was significantly higher in the IACL-R group. Osteophytes are a compensatory response to long-term mechanical joint instability[38]. At 3 months after the operation, the knee joint may have been mechanically stable, and the PTOA may have been at an early stage, so no obvious osteophyte formation was seen.

Some limitations of this study must be acknowledged. First, all imaging examinations were performed with the RH disengaged and in a non-weight-bearing state. In particular, X-ray examination cannot accurately reflect the progression of PTOA. Second, PTOA is a cartilage degenerative disease that can last for decades in humans[39]. However, all animals in our study were euthanized at 3 months after the surgery, which did not permit the assessment of the entire process of PTOA. Third, the state of tension of the ACL and the smaller motions of the joint in the IACL-R model cannot be detected using gait analysis.

\section{Conclusion}

In summary, the IACL-R model in the minipig reliably results in PTOA at 90 days after surgery, despite normalization of the gait parameters. This suggests that significantly elevated inflammatory factors may be a crucial factor in the pathogenesis of PTOA, and we hope that this will lead to the development of novel therapies for preventing PTOA.

\section{Abbreviations}

PTOA: post-traumatic osteoarthritis $₫ A C L$ : anterior cruciate ligament; IACL-R: idealized anterior cruciate ligament autograft reconstruction; IL: including interleukin ; TNF-a: tumor necrosis factor-a; GM-CSF: granulocyte-macrophage colony-stimulating factor $\mathrm{LH}$ : left hind foot; $\mathrm{RH}$ : right hind foot; $\mathrm{CT}$ : computed tomography $₫ 3 \mathrm{D}$ CT: three-dimensional computed tomography reconstruction; MRI :magnetic resonance imaging; Sag fs PD :Sagittal fat suppression proton density; WORMS: whole-organ magnetic resonance imaging score; MFC: medial femoral condyle; LFC: lateral femoral condyle; MTP: medial tibial plateau; LTP: lateral tibial plateau; GAGs: glycosaminoglycans; Col-II :type II collagen; MMP-13:matrix metalloproteinase; OARSI :Osteoarthritis Research Society International

\section{Declarations}

Ethics approval and consent to participate: 
This study did not need consent from any individuals/patients because no humans were involved in this study. All the procedures for animal surgery and pre- and post-operative care were approved by the Ethics Committee of the Second Hospital of Shanxi Medical University (Taiyuan, China; NO. 2017046).

\section{Consent for publication:}

Not applicable

\section{Availability of data and materials:}

The datasets used and or analysed during the current study are available from the corresponding author on reasonable request

\section{Competing interests:}

The authors declare that they have no competing interests

\section{Funding:}

The project was funded by the International Science and Technology Cooperation Program of China [Grant No. 2015DFA33050]

\section{Authors' contributions:}

RPZ participated in the study design, wrote the manuscript, performed most of the experiments, and analyzed data. PCL, ZQD, XCW conceived the study, revised the manuscript. HRW, YXY, FL carried out the part of imaging and histological assessment and helped to perform the statistical analysis. XDG, PFH, $\mathrm{ZL}, \mathrm{LAH}, \mathrm{AQL}$ performed the IACL-R of minipigs and gait Assessment. All authors read and approved the final manuscript.

\section{Acknowledgements:}

I would like to thank Yi xu and Kai Sun for providing assisting with imaging examination, and Yipeng Xue, veterinarian ,(Sciences of ShanXi Agricultural Academy Institute of Animal Husbandry\&Veterinary) for teaching us the animal management.

\section{References}

1. JM C, VG C, GJ S: a2-Macroglobulin: Autologous Protease Inhibition Technology. Physical Medicine \& Rehabilitation Clinics of North America 2016, 27(4):909-918.

2. Bjm B, Sari P, Adel S, Robert B, Louise N, Karl E: Increased risk of osteoarthritis after anterior cruciate ligament reconstruction: a 14-year follow-up study of a randomized controlled trial. Am J Sports Med 2014, 42(5):1049-1057. 
3. Björnsson H, Samuelsson K, Sundemo D, Desai N, Sernert N, Rostgård-Christensen L, Karlsson J, Kartus J: A Randomized Controlled Trial With Mean 16-Year Follow-up Comparing Hamstring and Patellar Tendon Autografts in Anterior Cruciate Ligament Reconstruction. American Journal of Sports Medicine 2016, 44(9): 2304-13.

4. Harris KP, Driban JB, Sitler MR, Cattano NM, Balasubramanian E, Hootman JM: Tibiofemoral Osteoarthritis After Surgical or Nonsurgical Treatment of Anterior Cruciate Ligament Rupture: A Systematic Review. Journal of Athletic Training 2015, 52(6):507.

5. Logerstedt D, Arundale A, Lynch A, Snyder-Mackler L: A conceptual framework for a sports knee injury performance profile (SKIPP) and return to activity criteria (RTAC). Brazilian Journal of Physical Therapy 2015, 19(5):340-359.

6. Han PF, Wei L, Duan ZQ, Zhang ZL, Chen TY, Lu JG, Zhao RP, Cao XM, Li PC, Lv Z: Contribution of IL$1 \beta, 6$ and TNF- $a$ to the form of post-traumatic osteoarthritis induced by "idealized" anterior cruciate ligament reconstruction in a porcine model. International Immunopharmacology. 2018;65:212-220

7. Rashid MH, Theberge Y, Elmes SJ, Perkins MN, Mcintosh F: Pharmacological validation of early and late phase of rat mono-iodoacetate model using the Tekscan system. European Journal of Pain 2013, 17(2):210-222.

8. Ruan MZC, Patel RM, Dawson BC, Jiang MM, Lee BHL: Pain, motor and gait assessment of murine osteoarthritis in a cruciate ligament transection model. Osteoarthritis Cartilage 2013, 21(9):13551364.

9. Shaw KE, Charlton JM, CkI P, de Vries CM, Redekopp MJ, White JA, Hunt MA: The effects of shoeworn insoles on gait biomechanics in people with knee osteoarthritis: a systematic review and metaanalysis. British Journal of Sports Medicine 2017:bjsports-2016-097108.

10. Suzuki K, Takahama M: Gait patterns of the diseased knee joint. Nihon Seikeigeka Gakkai Zasshi 1979, 53(7):847.

11. Wei L, Fleming BC, Sun X, Teeple E, Wu W, Jay GD, Elsaid KA, Luo J, Machan JT, Chen Q: Comparison of differential biomarkers of osteoarthritis with and without posttraumatic injury in the Hartley guinea pig model. J Orthop Res 2010, 28(7):900-906.

12. Demehri S, Guermazi A, Kwoh CK: Diagnosis and Longitudinal Assessment of Osteoarthritis: Review of Available Imaging Techniques. Rheumatic Disease Clinics of North America 2016, 42(4):607-620.

13. Kellgren JH, Lawrence JS: Radiological Assessment of Osteo-Arthrosis. Annals of the Rheumatic Diseases 1957, 16(4):494-502.

14. Holland JC, Brennan O, Kennedy OD, Mahony NJ, Rackard S, O'Brien FJ, Lee TC: Examination of osteoarthritis and subchondral bone alterations within the stifle joint of an ovariectomised ovine model. J Anat 2013, 222(6):588-597.

15. Peterfy CG, Guermazi A, Zaim S, Tirman PFJ, Miaux Y, White D, Kothari M, Lu Y, Fye K, Zhao S: WholeOrgan Magnetic Resonance Imaging Score (WORMS) of the knee in osteoarthritis. Osteoarthritis Cartilage 2004, 12(3):177-190. 
16. Wang XZ, Zheng YX, Cao YL, Xin-Feng GU, Wei SP, Gao NY, Liu T, Jian P: Study on the diagnostic value of whole-organ magnetic resonance imaging score(WORMS) in knee osteoarthritis. China Journal of Orthopaedics \& Traumatology 2012, 25(5):364-368.

17. Little CB, Smith MM , Cake MA, Read R.A , Murphy M.J , Barry F.P. The OARSI histopathology initiative e recommendations for histologicalassessments of osteoarthritis in sheep and goats. Osteoarthritis Cartilage. 2010;18:S80-S92

18. Wang S, Wei X, Zhou J, Zhang J, Li K, Chen Q, Terek R, Fleming BC, Goldring MB, Ehrlich MG: Identification of a2-macroglobulin as a master inhibitor of cartilagedegrading factors that attenuates the progression of posttraumatic osteoarthritis. Arthritis \& Rheumatology 2014, 66(7):1843-1853.

19. Barton KI, Shekarforoush M, Heard BJ, Sevick JL, Vakil P, Atarod M, Martin CR, Achari Y, Hart DA, Frank CB: Use of pre-clinical surgically induced models to understand biomechanical and biological consequences of PTOA development. Journal of Orthopaedic Research Official Publication of the Orthopaedic Research Society 2016, 35(3):454.

20. Dias IR, Viegas CA, Carvalho PP: Large Animal Models for Osteochondral Regeneration. Advances in Experimental Medicine \& Biology 2018, 1059:441.

21. Fang X, Mou Y, Huang Z, Li Y, Han L, Zhang Y, Feng Y, Chen Y, Jiang X, Zhao W: The sequence and analysis of a Chinese pig genome. GigaScience, 1,1(2012-11-15) 2012, 1(1):16.

22. Jeroen A, Steven B, Geert L, Jan D: Interspecies differences in bone composition, density, and quality: potential implications for in vivo bone research. Endocrinology 1998(2):663.

23. KãdÃđB MJ, Gwynn IA, NãqTzli HP: Collagen fibre arrangement in the tibial plateau articular cartilage of man and other mammalian species. Journal of Anatomy 1998, 193(1):23-34.

24. Preventive effects of hyaluronan from deterioration of gait parameters in surgically induced mice osteoarthritic knee model. Osteoarthritis and Cartilage 2014, 22(6) $ه 831-5$.

25. Capin JJ, Khandha A, Zarzycki R, Manal K, Buchanan TS, Snyder-Mackler L: Gait Mechanics After ACL Reconstruction Differ According to Medial Meniscal Treatment. Jbjs 2018, 100(14):1209-1216.

26. Hughes-Oliver CN, Srinivasan D, Schmitt D, Queen RM: Gender and Limb Differences in Temporal Gait Parameters and Gait Variability in Ankle Osteoarthritis. Gait \& Posture 2018, 65: 228-233.

27. Zahoor T, Mitchell R, Bhasin P, Schon L, Zhang Z: A Surgical Model of Posttraumatic Osteoarthritis With Histological and Gait Validation. Orthopaedic Journal of Sports Medicine,4,7(2016-7-28) 2016, 4(7).

28. Hart HF, Collins NJ, Ackland DC, Cowan SM, Hunt MA, Crossley KM: Immediate Effects of a Brace on Gait Biomechanics for Predominant Lateral Knee Osteoarthritis and Valgus Malalignment After Anterior Cruciate Ligament Reconstruction. American Journal of Sports Medicine 2016: 865-73

29. Pamukoff DN, Montgomery MM, Holmes SC, Moffit TJ, Garcia SA, Vakula MN: Association between gait mechanics and ultrasonographic measures of femoral cartilage thickness in individuals with ACL reconstruction. Gait \& Posture. 2018;65:221-227 
30. Mendias CL, Lynch EB, Davis ME, Sibilsky Enselman ER, Harning JA, Dewolf PD, Makki TA, Bedi A: Changes in circulating biomarkers of muscle atrophy, inflammation, and cartilage turnover in patients undergoing anterior cruciate ligament reconstruction and rehabilitation. American Journal of Sports Medicine 2013, 41(8):1819-1826.

31. Tiku K, Thakker-Varia S, Ramachandrula A, Tiku ML: Articular chondrocytes secrete IL-1, express membrane IL-1, and have IL-1 inhibitory activity. Cellular Immunology 1992, 140(1):1-20.

32. Chabane N, Zayed N, Afif H, Mfuna-Endam L, Benderdour M, Boileau C, Martel-Pelletier J, Pelletier JP, Duval N, Fahmi H: Histone deacetylase inhibitors suppress interleukin-1 $\beta$-induced nitric oxide and prostaglandin E2 production in human chondrocytes. Osteoarthritis \& Cartilage 2008, 16(10):12671274.

33. Suh HJ, Lee H, Min BJ, Jung SU, Jung EY: Effects of gangliosides from deer bone extract on the gene expressions of matrix metalloproteinases and collagen type II in interleukin-1 $\beta$-induced osteoarthritic chondrocytes. Nutrition Research \& Practice 2016, 10(6):569-574.

34. Burr DB, Schaffler MB: The involvement of subchondral mineralized tissues in osteoarthrosis: quantitative microscopic evidence. Microsc Res Tech 2015, 37(4):343-357.

35. Sniekers $\mathrm{YH}$, Weinans $\mathrm{H}$, Osch... GJv: Oestrogen is important for maintenance of cartilage and subchondral bone in a murine model of knee osteoarthritis. Arthritis research \& therapy 2010, 12(5):R182.

36. Murray MM, Fleming BC: Use of a bioactive scaffold to stimulate anterior cruciate ligament healing also minimizes posttraumatic osteoarthritis after surgery. American Journal of Sports Medicine 2013, 41(8):1762-1770.

37. Ryu YJ, Hong SH, Kim H, Choi JY, Yoo HJ, Kang Y, Park SJ, Kang HS: Fat-suppressed T2 mapping of femoral cartilage in the porcine knee joint: A comparison with conventional T2 mapping. Journal of Magnetic Resonance Imaging 2016, 45(4); 1076-81.

38. Kizaki K, Uchida S, Yamashita F, Tsukamoto M, Azuma K: Correction to: Microstructure of osteophytes in medial knee osteoarthritis. Clinical Rheumatology 2018, 37(10): 37:2879.

39. Jones MH, Spindler KP: Risk factors for Radiographic Joint Space Narrowing and Patient Reported Outcomes of Post-Traumatic Osteoarthritis after ACL Reconstruction: Data from the MOON Cohort. Journal of Orthopaedic Research 2017, 35(7):1366-1374.

\section{Tables}

Table 1. Kellgren-Lawrence grades on X-ray examination at 3 months $(n=7)$

\begin{tabular}{|c|c|c|c|c|c|c|}
\hline Group & Grade 0 & Grade [ & Grade [ & Grade [ & Grade [ & $\mathbf{P}$ \\
\hline Sham & 3 & 3 & 1 & 0 & 0 & \multirow[t]{2}{*}{0.132} \\
\hline IACL-R & 0 & 2 & 3 & 2 & 0 & \\
\hline
\end{tabular}


Table 2. WORMS, macroscopic cartilage score, osteophyte score and microscopic cartilage score,

\begin{tabular}{|llll|}
\hline Parameter & Sham & IACL-R & Sham/IACL-R \\
\hline WORMS sum (0-12) & Median (Q1, Q3) & Median (Q1, Q3) & P value \\
\hline MFC score (0-6) & $2(2,3.5)$ & $4(3.5,5.5)$ & 0.004 \\
\hline MTP score (0-6) & $1(1,2)$ & $2.5(2,3)$ & 0.015 \\
\hline Macroscopic OARSI score (0-20) & $4(3,7)$ & $2(1,2.5)$ & 0.049 \\
\hline MFC score (0-4) & $1(1,2)$ & $8(10,12)$ & 0.005 \\
\hline LFC score (0-4) & $1(0,1)$ & $1(1,2)$ & 0.008 \\
\hline MTP score (0-4) & $1(1,2)$ & $2(2,2)$ & 0.023 \\
\hline LTP score (0-4) & $1(0,10)$ & $1(1,2)$ & 0.038 \\
\hline Trochlea score (0-4) & $1(1,1)$ & $3(2,3)$ & 0.036 \\
\hline Osteophyte OARSI score (0-15) & $1(1,1)$ & $3(3,3)$ & 0.003 \\
\hline MFC score (0-4) & $0(0,1)$ & $1(0,1)$ & 0.009 \\
\hline LFC score (0-4) & $0(0,1)$ & $0(0,1)$ & 0.275 \\
\hline MTP score (0-4) & $0(0,1)$ & $0(0,1)$ & 0.645 \\
\hline LTP score (0-4) & $0(0,0)$ & $0(0,1)$ & 0.645 \\
\hline Trochlea score (0-4) & $0(0,1)$ & $1(1,2)$ & 0.591 \\
\hline Microscopic 0ARSI score (0-23) & $3(2,5)$ & $10(7,14)$ & 0.004 \\
\hline Structure score (0-10) & $2(1,3)$ & $4(3,6)$ & 0.027 \\
\hline Chondrocyte density score (0-4) & $0(0,1)$ & $1(1,2)$ & 0.018 \\
\hline Cell cloning score (0-4) & $0(0,1)$ & $1(1,2)$ & 0.031 \\
\hline GAG score (0-4) & $1(0,1)$ & $2(1,3)$ & $1(0,2)$ \\
\hline Demark score (0-3) & $0(0,1)$ & & 0.072 \\
\hline
\end{tabular}

Data are presented as median (Q1, Q3), $\mathrm{n}=7$.

MFC, medial femoral condyle; LFC, lateral femoral condyle; MTP, medial tibial plateau; LTP, lateral tibial plateau; GAG, glycosaminoglycan 


\section{Figures}
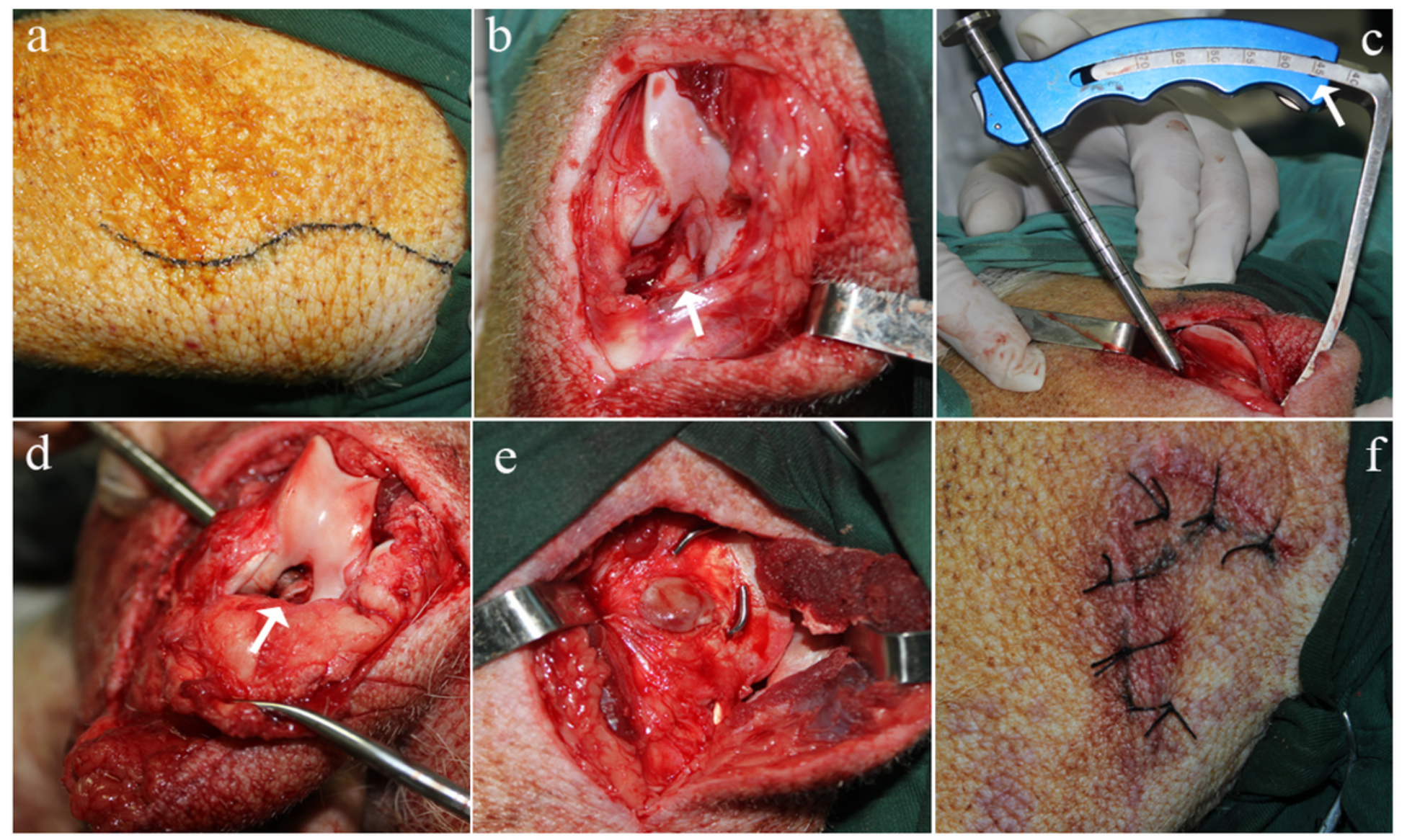

\section{Figure 1}

The surgical procedure performed on the right hind limb of a minipig in the IACL-R group. a: An S-shaped incision was made across the knee joint. b: The ACL was exposed (arrow). c: The ACL reconstruction guide was positioned at a $45^{\circ}$ angle (arrow) to the longitudinal axis of the femur. $\mathrm{d}$ : The tendon-bone segment was completely freed (arrow). e: The tendon-bone segment was fixed in situ with two crossed Kirschner wires. f: The incision was sutured layer by layer 

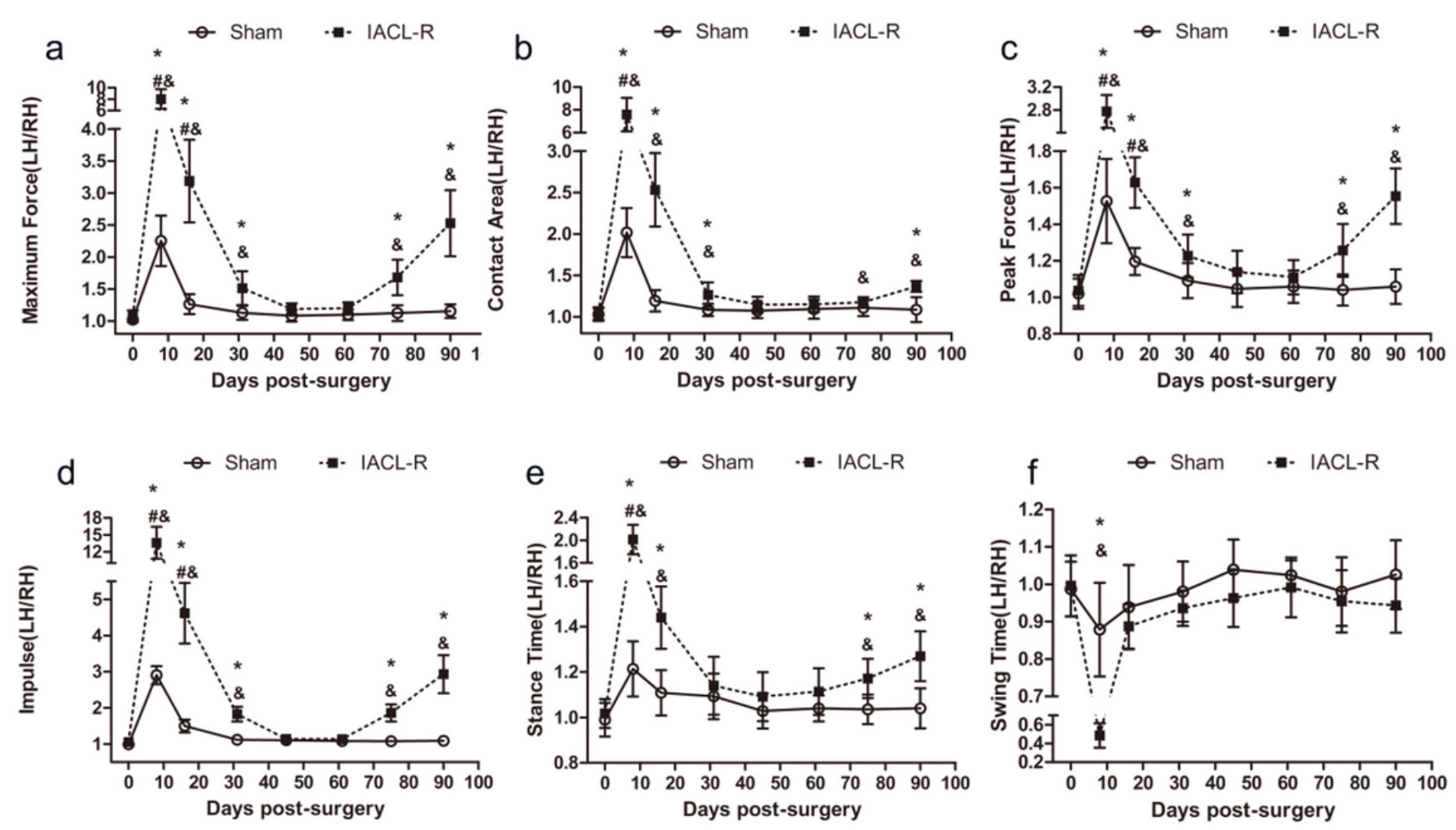

Figure 2

Line charts of gait analysis. a: Maximum force; b: Contact area; c: Peak force; d: Impulse; e: Stance time; $\mathrm{f}$ : Swing time * indicates significant differences between the sham and IACL-R groups at the same time point $(P<0.05)$. \# indicates significant differences between the preoperative (day 0$)$ and postoperative values (days $8,16,31,45,61,75$, and 90$)$ in the sham group $(P<0.05)$. \& indicates significant differences between the preoperative (day 0 ) and postoperative values (days $8,16,31,45,61,75$, and 90 ) in the IACLR group $(P<0.05)$. The bars show the mean \pm SD $(n=7)$. 

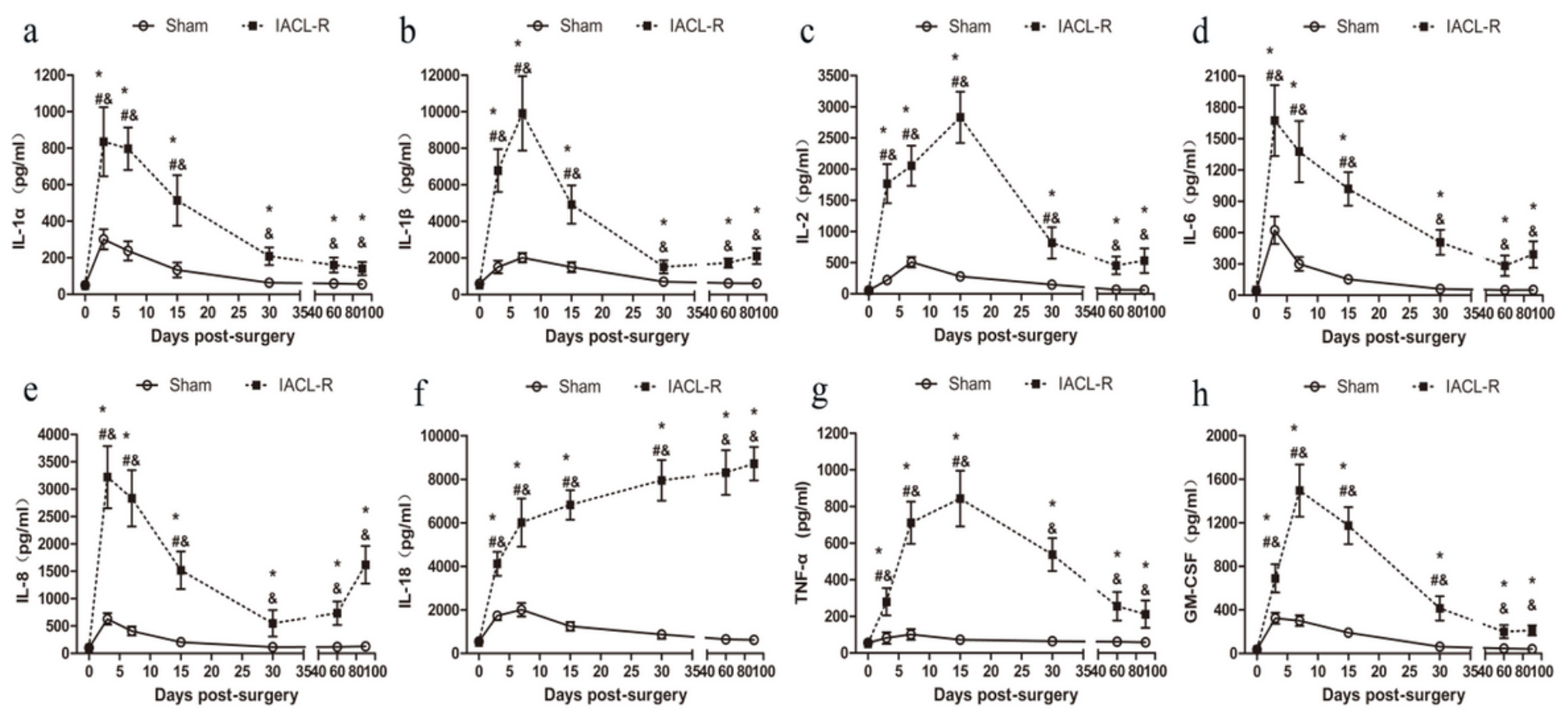

Figure 3

Line charts of Inflammatory factors in synovial fluid. a: IL-1a; b: IL-1ß; c: IL-2; d: IL-6; e: IL-8; f: IL-18; g: TNF-a; h: GM-CSF * indicates significant differences between the sham and IACL-R groups at the same time point $(P<0.05)$. \# indicates significant differences between the preoperative (day 0$)$ and postoperative values (days $3,7,15,30,60$ and 90 ) in the sham group $(P<0.05)$. \& indicates significant differences between the preoperative (day 0 ) and postoperative values (days $3,7,15,30,60$ and 90 ) in the IACL-R group $(P<0.05)$. The bars show the mean \pm SD $(n=7)$

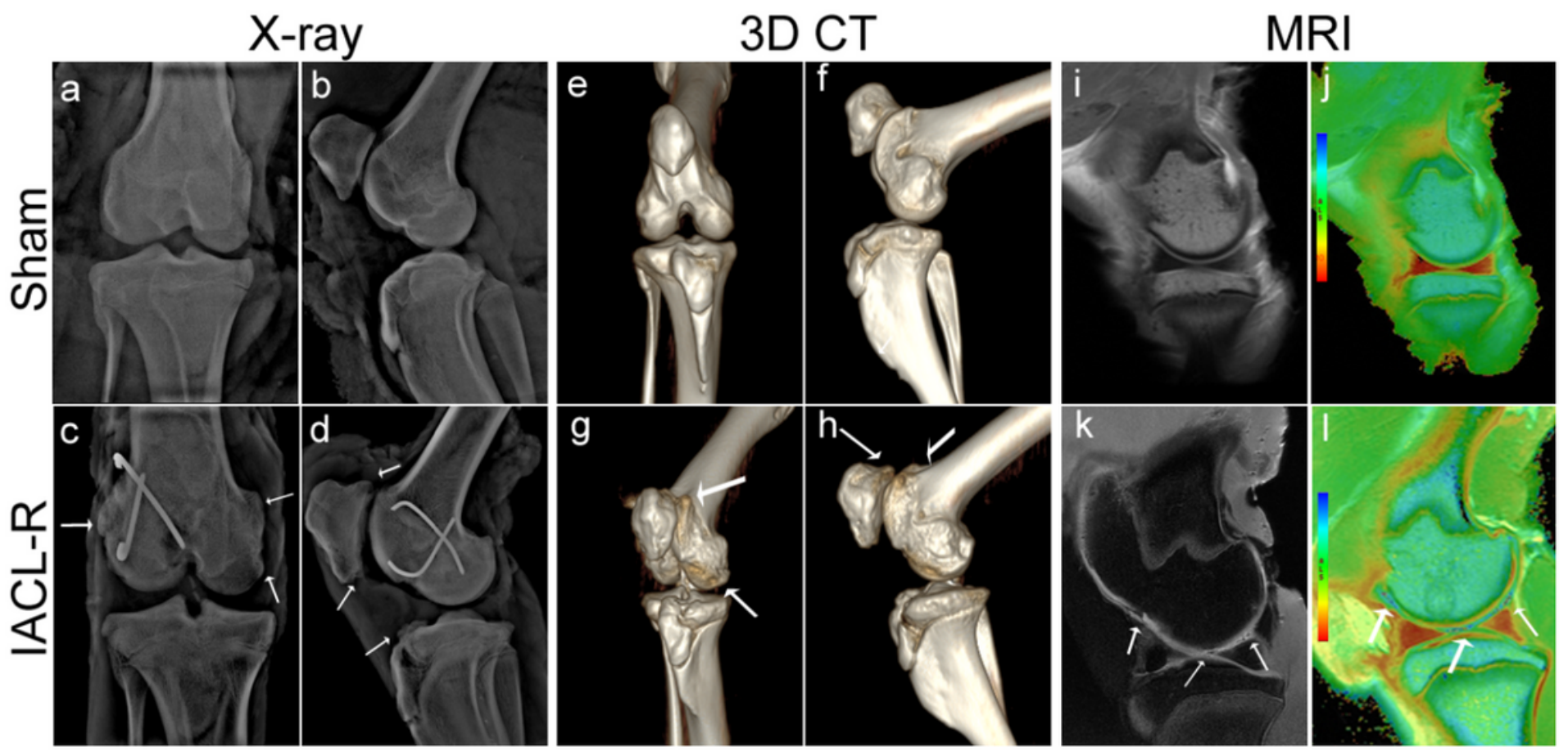




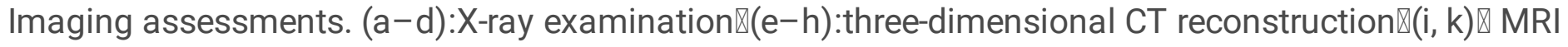
OSag fs PD of MFC and MTPQ (j, I): MRI OSag T2MAP of MFC and MTP. MFC, medial femoral condyle; MTP, medial tibial plateau. The white arrows indicate irregularities, osteophytes, and cartilage defects.

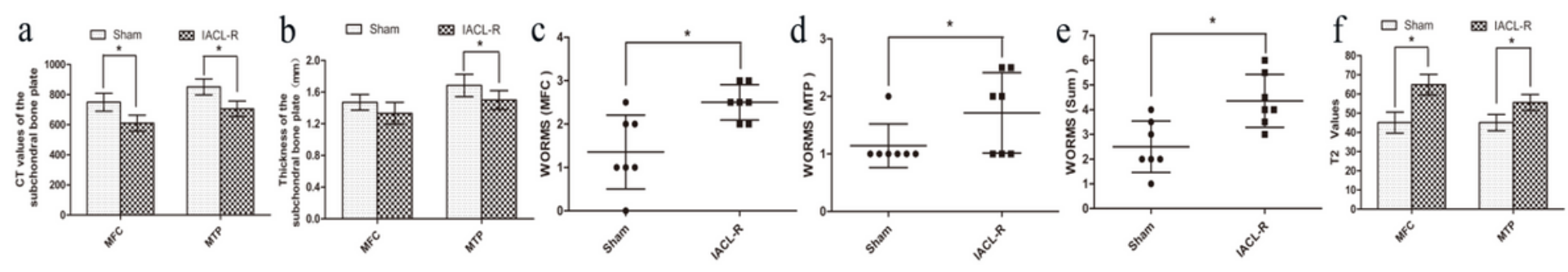

\section{Figure 5}

a:The CT values of the subchondral bone plate. sham group (MFC,749.17 \pm 55.46 ; MTP, $850.79 \pm 48.94$ ) ;IACL-R group (MFC, $610.22 \pm 49.50 ;$ MTP, $705.72 \pm 47.45)$. b: The thickness of the subchondral bone plate. sham group (MFC, 1.47 $\pm 0.09(\mathrm{~mm}) ;$ MTP, $1.68 \pm 0.13(\mathrm{~mm})$ ) ;IACL-R group (MFC, $1.33 \pm 0.12(\mathrm{~mm})$; MTP, $1.50 \pm 0.11(\mathrm{~mm})$ ). (c-e) Quantification of the MRI OSag fs PD results using the WORMS guidelines. $f: Q u a n t i f i c a t i o n$ of the T2 values obtained using MRI sag T2MAP. Sham group( MFC, 45.08 \pm 5.06 ; MTP, $44.80 \pm 4.18$ ); IACL-R group( MFC, $64.85 \pm 4.95 ;$ MTP, $55.6 \pm 3.83$ ). MFC, medial femoral condyle; MTP, medial tibial plateau. The bars show the mean \pm SD. ${ }^{*}=P<0.05$.

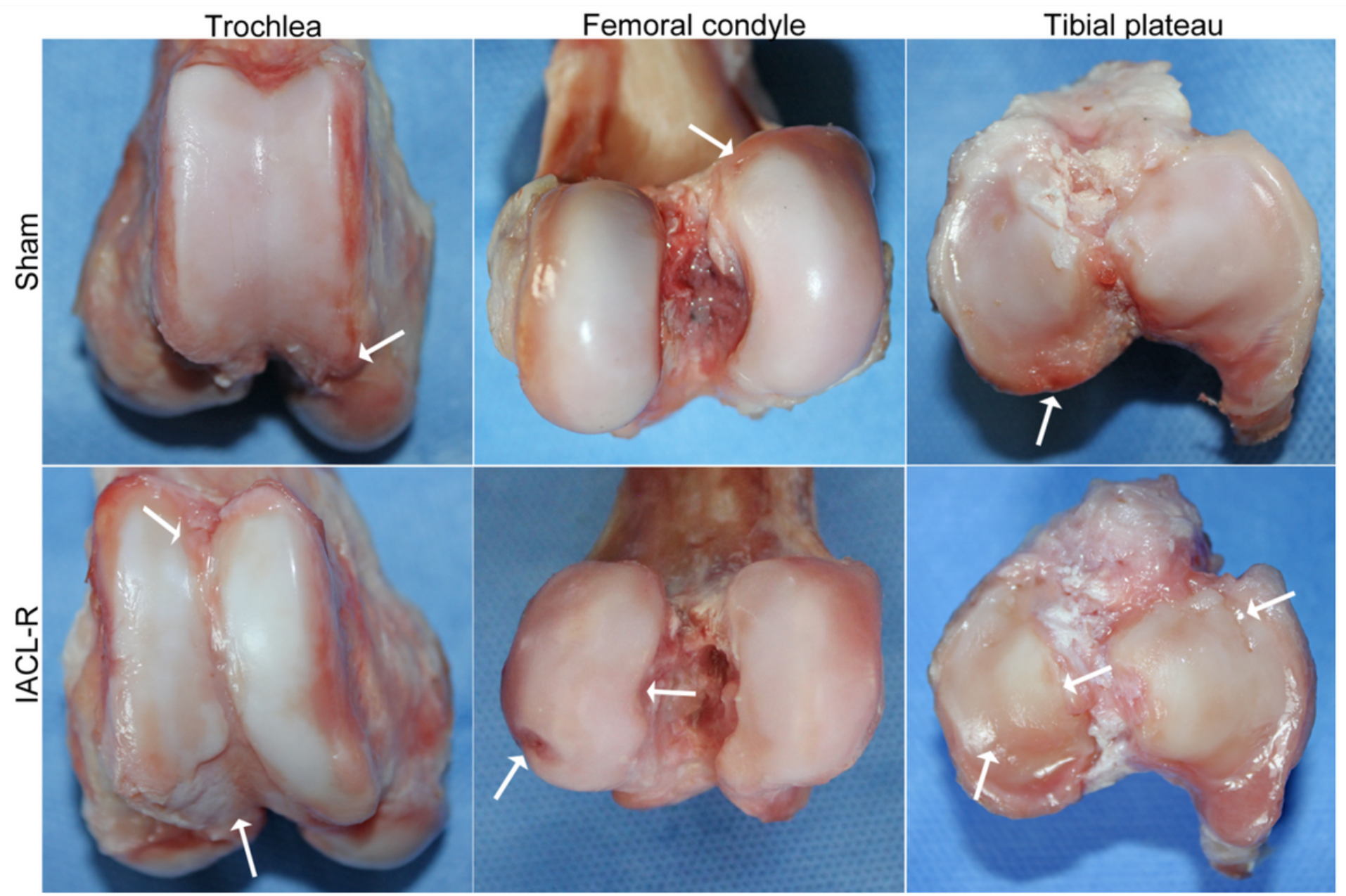


Images of the trochlea, femoral condyle, and tibial plateau for the macroscopic cartilage and osteophyte assessment according to OARSI guidelines. The white arrows indicate cartilage damage, irregularities, and osteophytes.
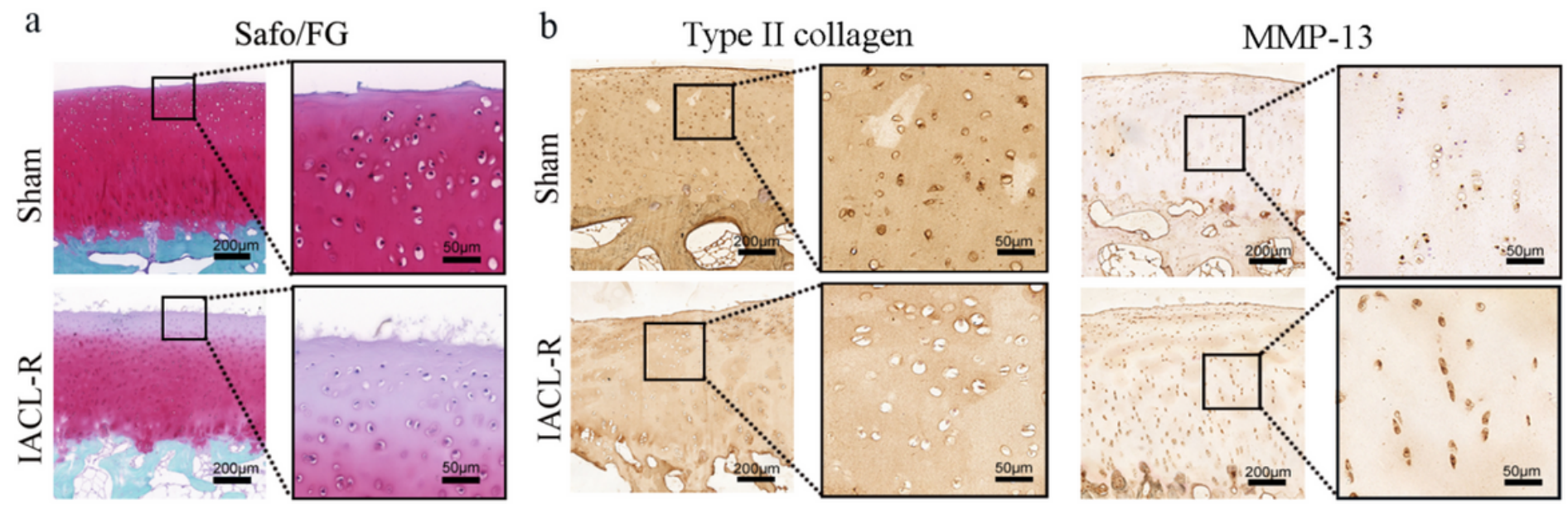

\section{Figure 7}

a: Slight decrease in safranin 0 staining and slight surface fibrillation are seen in the sham group as compared with the IACL-R group. b: Type II collagen expression was higher in the sham group than in the IACL-R group. In contrast, matrix metalloproteinase-13 (MMP-13) expression was lower in the sham group than in the IACL-R group. The right panels show higher-magnification views of the boxed areas in the left panels.

\section{Supplementary Files}

This is a list of supplementary files associated with this preprint. Click to download.

- AdditionalTable1.docx

- AdditionalFigure12.docx

- AdditionaltextAB.docx 